\title{
Dinâmica da infecção natural por Eimeria spp. em cordeiros da raça Santa Inês criados em sistema semi-intensivo no Norte de Minas Gerais
}

\author{
[Dinamics of naturally Eimeria spp. infected Santa Inês lambs reared under semi-intensive \\ conditions in Northern Minas Gerais, Brazil] \\ T.P. Silva, E.J. Facury Filho* ${ }^{*}$ A.B.V. Nunes, F.H.M.A.R. Albuquerque, \\ P.M. Ferreira, A.U. Carvalho \\ Escola de Veterinária - UFMG \\ Caixa Postal 567 \\ 30123-970 - Belo Horizonte, MG
}

\begin{abstract}
RESUMO
Primo-infecção por Eimeria spp., evolução da infecção e principais espécies presentes foram avaliadas em 30 cordeiros da raça Santa Inês, machos, lactentes, criados em sistema semi-intensivo no Norte de Minas Gerais. A detecção inicial de oocistos nas fezes ocorreu entre o $16^{\circ}$ e o $32^{\circ}$ dias de idade e a espécie mais freqüente foi E. ovinoidalis (52,8\%). A excreção de oocistos até a $16^{\mathrm{a}}$ semana de idade caracterizou-se por aumento gradual a partir da segunda semana, com picos na sétima e na $10^{\text {a }}$ semanas. O segundo pico foi mais baixo que o primeiro, com queda mais persistente, indicando o desenvolvimento de imunidade. Foram identificadas 11 espécies de Eimeria: E. parva, E. ovinoidalis, E. crandallis, E. caprovina, E. ovina, E. ahsata, E. pallida, E. faurei, E. intrincata, E. granulosa e E. punctata. E. crandallis foi a mais freqüente $(47,2 \%)$. As condições locais e de manejo, apesar de consideradas adversas, foram propícias à esporulação de oocistos de Eimeria spp. e à constante reinfecção dos cordeiros.
\end{abstract}

Palavras-chave: cordeiro lactente, coccidiose, eimeriose, Eimeria spp., OOPG

\begin{abstract}
Eimeria spp. primoinfection, the development of this infection and the infecting species were determined by the evaluation of 30 Santa Inês lactating male lambs reared under semi-intensive conditions in Northern Minas Gerais. Oocysts were first detected in faeces of lambs between the $16^{\text {th }}$ and the $32^{\text {th }}$ week of age. The most frequent species was E. ovinoidalis (52.8\%). The oocysts output until the $16^{\text {th }}$ week of age was characterized by peaks at the $7^{\text {th }}$ and $10^{\text {th }}$ weeks. The second peak was lower than the first, followed by a subsequent reduction more persistent than the first one, suggesting the development of immunity. Eleven species of Eimeria were identified: E. parva, E. ovinoidalis, E. crandallis, E. caprovina, E. ovina, E. ahsata, E. pallida, E. faurei, E. intrincata, E. granulosa and E. puncata. E. crandallis was the most frequent (47.2\%). Although considered adverse, the environmental conditions allowed the sporulattion of Eimeria spp. oocysts and the constant reinfection of the lambs.
\end{abstract}

Keywords: lactating lamb, coccidiosis, eimeriosis, Eimeria spp., OOPG

\section{INTRODUÇÃO}

Como em todo sistema de produção, a criação de ovinos tem alguns pontos de estrangulamento importantes e que devem ser bem conhecidos e controlados para otimizar e tornar essa atividade viável.
A coccidiose, doença infecciosa causada por protozoários do gênero Eimeria, tem grande importância econômica na ovinocultura (Foreyt, 1990; Gjerde e Helle, 1991). Está presente em todos os sistemas de produção e ganha maior importância diante da intensificação da criação. (Catchpole et al., 1993; Urquhart et al., 1998).

Recebido em 28 de março de 2007

Aceito em 17 de setembro de 2007

*Autor para correspondência (corresponding author)

E-mail: facury@vet.ufmg.br 
Consideráveis perdas econômicas resultam da doença clínica e, principalmente, da doença subclínica, causadora esta última de prejuízos que, muitas vezes, são ignorados pelo produtor, mas que são constantes e de grande importância.

A coccidiose subclínica determina reduções na taxa e na eficiência de ganho de peso e crescimento dos animais afetados, além de torná-los mais susceptíveis a outras doenças (Foreyt, 1990; Faizal et al., 1999). Morbidade, mortalidade e impacto econômico estão associados principalmente aos cordeiros jovens, responsáveis pela liberação de grandes quantidades de oocistos no ambiente.

Apesar da alta freqüência de eimeriose na criação de ovinos, há poucos estudos sobre o comportamento de Eimeria spp. e a freqüência de espécies nos rebanhos ovinos do Sudeste do Brasil e nenhum estudo no Norte de Minas Gerais. O objetivo deste trabalho foi determinar a primiinfecção de cordeiros por Eimeria spp., estudar a evolução da infecção e identificar as principais espécies em cordeiros lactentes em um rebanho no Norte de Minas Gerais.

\section{MATERIAL E MÉTODOS}

O experimento foi realizado no período de abril a julho de 2005 em uma fazenda na região de Montes Claros, na bacia do Alto-Médio São Francisco, Norte de Minas Gerais, no polígono da seca. O clima local é caracterizado como quente e seco, com temperaturas anuais médias entre $22^{\circ}$ e $24^{\circ} \mathrm{C}$ e precipitação total de 1000 a $1200 \mathrm{~mm} /$ ano. A vegetação é caracterizada pela transição entre o cerrado e a caatinga.

A área da fazenda destinada à criação de ovinos para corte é de 5 ha. O rebanho, composto por 350 matrizes da raça Santa Inês, é criado em sistema de pastejo contínuo em pastos de braquiarão e Cynodon spp. $\mathrm{Na}$ seca, os animais recebem suplementação de volumoso e concentrado no cocho. Sal mineral específico para ovinos é fornecido à vontade.

Em novembro de 2004, adotou-se a estação de monta de oito semanas com todas as matrizes, que resultou em nascimentos de abril a junho de 2005 . Cordeiros e suas mães foram confinados até meados de maio, quando se construiu um creepfeeding para os cordeiros; as matrizes, paridas a mais de 15 dias, passaram a ser soltas no pasto durante o dia, mas os cordeiros permaneceram confinados em tempo integral.
Ao final da época de nascimentos, o curral maior alojava 300 cordeiros com diferenças de idade de até dois meses e, à noite, aproximadamente mais 250 ovelhas em lactação. A desmama, tradicionalmente programada para ocorrer aos 90 dias de idade, não havia sido efetivada até o término do experimento. Não houve interferência alguma no manejo da fazenda e os cordeiros avaliados permaneceram junto aos demais, sob as mesmas condições do rebanho.

Amostras de fezes de 30 cordeiros machos da raça Santa Inês, com idades aproximadas e nascidos de parto simples foram coletadas, diariamente, do nascimento aos 32 dias de idade para determinar a primeira detecção de oocistos nas fezes, que caracterizam a primo-infecção. Uma vez constatado o aparecimento de oocistos nas fezes, as coletas passavam a ser semanais, o que se seguiu até o final do experimento, que ocorreu na $16^{\mathrm{a}}$ semana de vida. Semanalmente, de todos os animais examinados clinicamente, coletavam-se amostras de fezes.

As fezes foram avaliadas pela técnica de flutuação em solução saturada de açúcar (Ueno e Gonçalves, 1998). As amostras positivas, analisadas quantitativamente pelo método de contagem de oocistos por grama de fezes (OOPG), de acordo com a técnica de Gordon e Whitlock, modificada, segundo Ueno e Gonçalves (1998), eram colocadas para esporular em placas de petri com solução de bicromato de potássio a $2,5 \%$ por sete dias, em temperatura ambiente, para posterior identificação das espécies de Eimeria. Procedeu-se à diferenciação de espécies de acordo com forma, cor, presença ou ausência de micrópila e capuz micropilar, e pelo tamanho dos oocistos, além das características morfológicas dos esporocistos, seguindo os parâmetros de Vercruysse (1982), Amarante e Barbosa (1992), e fotografias e descrições de oocistos de ovinos de Catchpole et al. (1975), Levine (1985), Reginsson e Richter (1997) e Kaya (2004).

A espécie E. caprovina, identificada neste estudo, foi erroneamente classificada como E. ovinoidalis. Dessa forma, optou-se por classificar as duas espécies em um só grupo, doravante denominado $E$. ovinoidalis/E. caprovina.

\section{RESULTADOS E DISCUSSÃO}

Os cordeiros começaram a eliminar oocistos nas fezes entre 16 e 32 dias de vida, com maiores contagens no $22^{\circ}, 29^{\circ}$ e $30^{\circ}$ dias de idade. Estes 
resultados são semelhantes aos citados de Mason (1977), que detectou a presença de oocistos nas fezes de cordeiros entre o $19^{\circ}$ e $37^{\circ}$ dias de idade, e compatíveis com os de Chapman et al. (1973) e Pout (1976), que, em cordeiros criados em pasto, relataram o aparecimento de oocistos nas fezes a partir da segunda semana de idade. Taylor et al. (1973), em sistema de produção intensiva de cordeiros, observaram que a partir da terceira semana de idade todos os animais já excretavam oocistos nas fezes.

A infecção responsável pela primeira detecção de oocistos nas fezes ocorreu, segundo os períodos pré-patentes apresentados por Levine (1985), nos primeiros dias após o nascimento, precisamente nas duas primeiras semanas de idade, semelhantemente aos resultados de Vieira et al. (1999) em Sobral (CE). Provavelmente a infecção dos cordeiros ocorreu pela ingestão de oocistos presentes na teta das mães, por eventual ingestão de terra (Helle, 1971; Pout, 1973), pelo ato de lamber o próprio pêlo ou pelo contato com bebedouros contaminados por oocistos.

No $16^{\circ}$ e $17^{\circ}$ dias de idade predominou a eliminação de oocistos de E. ovinoidalis/E. caprovina. Essa foi a espécie mais freqüente na primi-infecção $(52,8 \%)$, seguida por $E$. faurei $(25,9 \%)$, E. crandallis $(11,4 \%)$, E. ovina $(4,6 \%), E$. intrincata $(3,0 \%)$, E. ahsata $(1,2 \%)$, E. pallida $(0,5 \%)$, E. parva $(0,4 \%)$ e E. granulosa $(0,2 \%)$.

A partir do $18^{\circ}$ dia de idade as infecções tornaramse mistas, e variaram entre duas e sete espécies por amostra, à exceção do $25^{\circ}$ e $27^{\circ}$ dias, quando predominou a excreção de oocistos de $E$. ovinoidalis/E. caprovina. Ao todo foram identificadas nove espécies de Eimeria até o $30^{\circ}$ dia de vida. Uma $10^{\mathrm{a}}$ espécie, E. punctata, foi identificada ao $42^{\circ}$ dia, semelhantemente ao resultado obtido por Mason (1977). As espécies, suas freqüências e as idades nas quais foram observadas nas fezes pela primeira vez constam da Tab. 1.

Tab. 1. Idade (dias) da primeira eliminação de oocistos de Eimeria spp., em fezes de cordeiros criados em sistema semi-intensivo no Norte de Minas, idade provável de infecção e freqüência de animais infectados, por espécie de Eimeria, entre abril e julho de 2005

\begin{tabular}{|c|c|c|c|c|c|}
\hline \multirow[b]{2}{*}{ Espécie } & \multicolumn{3}{|c|}{ Idade (dias) } & \multirow[b]{2}{*}{$\begin{array}{l}\text { Número de } \\
\text { cordeiros }\end{array}$} & \multirow[b]{2}{*}{$\begin{array}{l}\text { Freqüência de cordeiros } \\
\text { por espécie de Eimeria }\end{array}$} \\
\hline & $\begin{array}{l}\text { Primeira } \\
\text { detecção de } \\
\text { oocistos }\end{array}$ & Período pré-patente & $\begin{array}{l}\text { Idade provável } \\
\text { da infecção }\end{array}$ & & \\
\hline E. intrincata & 30 & $20-27$ & $3-10$ & 1 & 3,33 \\
\hline E. parva & $21-29$ & $7-10$ & $11-22$ & 2 & 6,67 \\
\hline E. pallida & $22-29$ & ------ & ---- & 2 & 6,67 \\
\hline E. ovina & $22-26$ & $19-29$ & $0-7$ & 2 & 6,67 \\
\hline E.crandallis & $20-32$ & $13-20$ & $0-19$ & 6 & 20,00 \\
\hline E. ahsata & $22-30$ & $18-21$ & $1-12$ & 2 & 6,67 \\
\hline E. ovinoidalis & $16-32$ & $9-15$ & $1-23$ & 24 & 80,00 \\
\hline E. faurei & $18-32$ & $14-18$ & $0-18$ & 11 & 36,67 \\
\hline E. granulosa & 22 & $14-17$ & $5-8$ & 1 & 3,33 \\
\hline
\end{tabular}

A idade à primeira detecção de oocistos de $E$. ovinoidalis/E. caprovina, E. faurei e E. crandallis demonstra a capacidade dessas espécies em infectar cordeiros jovens, aspecto já relatado por Gregory e Catchpole (1989) e Catchpole et al. (1993). Em condições de infecção natural, E. ovinoidalis/E. caprovina apareceu pela primeira vez quatro dias antes de $E$. crandallis, sugerindo que o período prépatente (PPP) de E. ovinoidalis/E. caprovina foi menor que o de E. crandallis. Segundo Levine (1985), o período pré-patente (PPP) de $E$. ovinoidalis varia de nove a 15 dias, enquanto que de E. crandallis de 13 a 20 dias. O PPP mínimo nas espécies é confirmado neste estudo. Gregory e Catchpole (1989) também observaram que o PPP de E. ovinoidalis é menor do que o de $E$. crandallis.

Após as primeiras eliminações, o comportamento de excreção caracterizou-se pelo aumento progressivo e relativamente rápido, dando origem a um primeiro pico à sétima semana de idade (125.985,71 OOPG), seguido de queda à oitava semana $(24.835,71$ OOPG) e novo pico, dessa vez mais baixo, (101.657,14 OOPG) à $10^{\mathrm{a}}$ semana de vida dos cordeiros, seguido por nova queda de eliminação de oocistos mais contínua e gradual do que a primeira (3.800 OOPG à $16^{\mathrm{a}}$ semana) (Fig. 1). Vieira et al. (1999), em estudo 
com cordeiros da raça Santa Inês em Sobral (Ceará), à semelhança do que foi observado no presente estudo, também observaram a eliminação de oocistos nas fezes a partir da terceira semana de vida, com um primeiro pico à sétima semana e um segundo, menor que o primeiro. Esses autores ainda observaram um terceiro pico à $20^{\mathrm{a}}$ semana de idade, menor ainda do que o segundo. A eliminação de oocistos por cordeiros, portanto, diminui com o avançar da idade dessa categoria de animais.

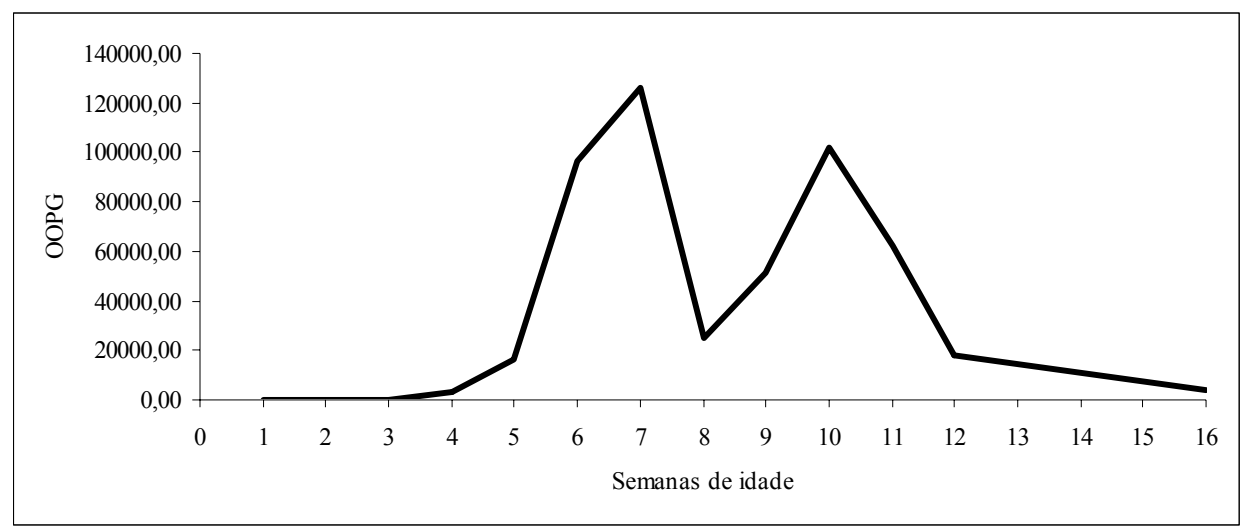

Figura 1. Distribuição das médias de OOPG em cordeiros lactentes naturalmente infectados por Eimeria spp., criados em sistema semi-intensivo no Norte de Minas Gerais, entre abril e julho de 2005.

O primeiro pico observado caracterizou-se pelo predomínio de E. crandallis (Tab. 2) e remete a uma infecção por oocistos de Eimeria spp. entre o $21^{\circ}$ e $35^{\circ}$ dias de vida.

A queda na excreção de oocistos logo após o primeiro pico, segundo Chapman et al. (1973), deve-se ao processo de desenvolvimento de imunidade conforme o avanço da idade dos cordeiros. No presente estudo, o desenvolvimento de imunidade após o primeiro pico refletiu-se em um segundo pico com menor contagem. $\mathrm{O}$ fato de haver esse segundo pico indica, também, que essa imunidade foi parcial.

O segundo pico teve predomínio de E. parva (Tab. 2) e possivelmente é o reflexo de uma infecção que ocorreu entre o $51^{\circ}$ e $59^{\circ}$ dias de idade. Logo após esse pico houve queda caracterizada por uma linha em franco declínio, mais gradual e persistente que a primeira, e com tendência à estabilização em baixas contagens de oocistos no final, quando da última coleta à $16^{\mathrm{a}}$ semana de vida. Essa queda reflete o desenvolvimento de uma imunidade mais efetiva.

Tabela 2. Distribuição das espécies de Eimeria em fezes de cordeiros lactentes, naturalmente infectados, criados em sistema semi-intensivo no Norte de Minas Gerais, entre abril a julho de 2005

\begin{tabular}{ccccccccccc}
\hline Idade & \multicolumn{10}{c}{ Espécie } \\
\cline { 2 - 11 } (semana) & E. intrincata & E. parva & E. pallida & E. crandallis & E. ovina & E. ahsata & E. ovinoidalis & E. faurei & E. granulosa & E. punctata \\
\hline 4 & 0 & 0 & 0 & 0 & 29,3 & 0 & 70,7 & 0 & 0 & 0 \\
5 & 0 & 35 & 0 & 35,7 & 0,1 & 0 & 19,7 & 9,5 & 0 & 0 \\
6 & 0,2 & 2,5 & 2,4 & 50,1 & 11,6 & 2 & 10,1 & 21 & 0 & 0,1 \\
7 & 0,7 & 2,1 & 2,1 & 50,7 & 6,2 & 14,8 & 19,1 & 4,3 & 0 & 0 \\
8 & 0,2 & 13,7 & 25,2 & 36,4 & 7,4 & 4,8 & 8,1 & 4,2 & 0 & 0 \\
9 & 0 & 23,8 & 2,9 & 55,1 & 4,7 & 3,5 & 3,5 & 6,2 & 0,3 & 0 \\
10 & 0,2 & 42 & 5,6 & 26,4 & 12,7 & 0,9 & 7,2 & 5 & 0 & 0 \\
11 & 0,5 & 50,4 & 0,4 & 24,8 & 16,6 & 1,5 & 2,4 & 3,4 & 0 & 0 \\
12 & 0,6 & 24,7 & 1,6 & 23,4 & 8,9 & 28,8 & 6,2 & 5,8 & 0 & 0 \\
\hline
\end{tabular}


Ao todo foram identificadas 11 espécies de Eimeria. A mais freqüente foi $E$. crandallis $(41,4 \%)$, seguida por E. parva $(19,3 \%), E$. ovinoidalis/E. caprovina, E. ovina, E. ahsata, E. pallida, E. faurei, E. intrincata, E. granulosa e E. puncata. Sete espécies foram comuns às encontradas por Amarante e Barbosa (1991) em Botucatu: E. crandallis, E. ovinoidalis, E. ovina, E. ahsata, E. pallida, E. intrincata e E. parva. As espécies $E$. faurei e $E$. punctata foram observadas em Porto Alegre por Silva et al. (1987/8), citado por Amarante e Barbosa (1991). Silva et al.
(1987/8) ainda encontraram outras espécies comuns às identificadas neste estudo, E. parva, $E$. crandallis, E. ahsata, E. ovina, E. ovinoidalis e E. intrincata. Vieira et al. (1999) verificaram nove espécies de Eimeria, todas comuns às encontradas no presente estudo, E. parva, E. granulosa, E. crandallis, E. ahsata, E. ovina, E. ovinoidalis, E. caprovina, E. faurei e E. intrincata. Na Fig. 2 estão fotografias de algumas espécies de Eimeria identificadas neste estudo.

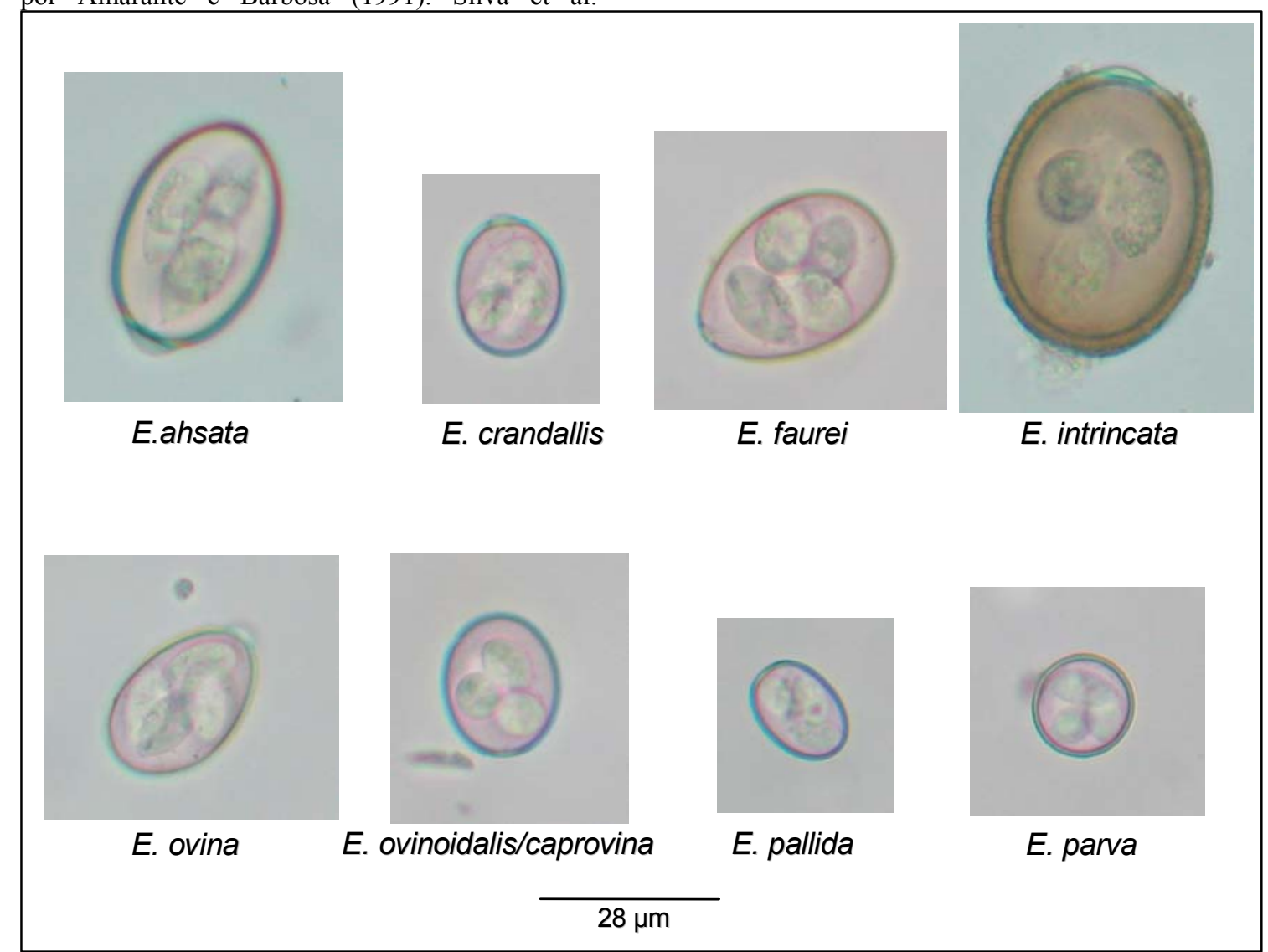

Figura 2. Oito espécies de Eimeria identificadas em fezes de cordeiros lactentes, naturalmente infectados, criados em sistema semi-intensivo no Norte de Minas Gerais entre abril a julho de 2005.

Ocorreram flutuações na porcentagem de espécies encontradas nas fezes dos cordeiros. Em geral, essas flutuações caracterizaram-se por dois picos seguidos de quedas subseqüentes. A queda observada após o segundo pico tendeu a ser mais consistente e efetiva, repetindo o comportamento descrito para excreção de oocistos (Tab. 2).

As condições ambientais e de manejo encontradas entre os meses de abril e julho de 2005, período de seca na fazenda deste estudo, localizada na região da bacia do Alto-Médio São Francisco, Norte de Minas Gerais, no polígono da seca, apesar de consideradas adversas, foram propícias à esporulação de oocistos de Eimeria spp. e à constante reinfecção dos animais, refletida na contínua eliminação de oocistos nas fezes dos cordeiros. Como não houve casos clínicos de coccidiose, a forma encontrada da doença foi subclínica. 


\section{REFERÊNCIAS BIBLIOGRÁFICAS}

AMARANTE, A.F.T.; BARBOSA, M.A. Species of coccidia occuring in lambs in São Paulo State, Brazil. Vet. Parasitol., v.41, p.189193, 1992.

CATCHPOLE, J.; NORTON, C.C.; GREGORY, M.W. Immunization of lambs against coccidiosis. Vet. Rec. v. 132, p.56-59, 1993.

CATCHPOLE, J.; NORTON, C.C.; JOYNER, L.P. The occurrence of Eimeria weybridgensis and other species of coccidia in lambs in England and Wales. Br. Vet. J., v.131, p.392-401, 1975.

CHAPMAN, H.D.; LEWIS, J.A.; SEARLE, R.M. The effect of naturally acquired infections of coccidian in lambs. Res. Vet. Sci., v.14, p.369$375,1973$.

FAIZAL，A.C. M.; RAJAPAKSE， R.P.V.J.; JAYASINGHE, S.R. et al. Prevalence of Eimeria spp. and gastrointestinal nematodes versus weight gains in treated goats raised in the dry areas of Sri Lanka. Small Rum. Res., v.34, p.21$25,1999$.

FOREYT, W.J. Coccidiosis and cryptosporidiosis in sheep and goats. Vet. Clin. N. Am.: Food Anim. Pract., v.6, p.655-670, 1990.

GJERDE, B.; HELLE, O. Chemoprophylaxis of coccidiosis in lambs with a single oral dose of toltrazuril. Vet. Parasitol., v.38, p.97-107, 1991.

GREGORY, M.W.; CATCHPOLE, J. Ovine coccidiosis: heavy infection in young lambs increases resistance without causing disease. Vet. Rec., v.124, p.458-461, 1989.

HELLE, O. Gastrointestinal parasites in sheep on lowland pastures in eastern Norway. Acta Vet. Scand., suppl. 34, p.1-118, 1971.

KAYA, G. Prevalence of eimeria species in lambs in Antakya province. Turk. J. Vet. Anim. Sci., v.28, p.687-692, 2004.
LEVINE, N.D. (Ed). Veterinary protozoology. Ames: Iowa State University, 1985. p.130-163.

MASON, P. Naturally acquired coccidia infection in lambs in Otago. N. Zeal. Vet. J., v.25, p.30-33, 1977.

POUT, D.D. Coccidiosis of lambs. I. Observations on the naturally acquired infection. Br. Vet. J., v.129, p.555-567, 1973.

POUT, D.D. Coccidiosis of sheep: a review. Vet. Rec., v.98, p.340-341, 1976.

REGINSSON, K.; RICHTER, S. Coccidia of the genus Eimeria in sheep in Iceland. Icel. Agric. Sci., v.11, p.99-106, 1997.

SILVA, N.R.S.; ARAUJO, F.A.P.; CHAPLIN, E.L. Eimerídios de ovinos no município de Porto Alegre. Arq. Fac. Vet. UFRGS, v.15-16, p.41-45, 1987/1988.

TAYLOR, S.M.; O'HAGAN, J.; MCCRACKEN, A. et al. Diarrhoea in intensively-reared lambs. Vet. Rec., v.93, p.461464, 1973.

UENO, H.; GONÇALVES, P.C. Manual para diagnóstico das helmintoses de ruminantes. 4.ed. Tokio: Japan International Cooperation Agency, 1998. p.14-15, 25-28.

URQUHART, G.M.; ARMOUR, J.; DUNCAN, J.L. et al. (Eds). Parasitologia veterinária. 2.ed. Rio de Janeiro: Guanabara Koogan, 1998. p.196202.

VERCRUYSSE, J. The coccidia of sheep and goats in Senegal. Vet. Parasitol. v.10, p.297306, 1982.

VIEIRA， L.S.; CAVALCANTE， A.C.R.; XIMENES, L.J.F. Infection with Eimeria species in hair sheep reared in Sobral, Ceará State, Brazil. Rev Med Vet, v.150, p.547-550, 1999. 